\title{
Liderazgo, comunicación organizacional y motivación de los trabajadores en la industria maquiladora de Nogales, Sonora
} Leadership, organizational communication and motivation
of workers in the maquiladora industry of Nogales, Sonora

Mirla Yessenia Castro Rodríguez*

Universidad de Sonora

Avenida UNISON núm. 343,

C.P. 84093, Nogales,

Sonora, México

Editor: Rogelio del Prado Flores

Fecha de recepción: 17 de agosto de 2020

Fecha de aceptación: 3 de noviembre de 2020 mirla.castro@unison.mx

https://orcid.org/0000-0003-4707-7577

\section{RESUMEN}

Actualmente existe una gran rotación de personal dentro de la zona maquiladora de la frontera de Nogales, Sonora. La mirada se enfoca en identificar la percepción que se tiene dentro de la mano operativa de la zona maquiladora, y cómo la comunicación organizacional, el liderazgo y la posición dentro de la estructura organizacional influyen en la decisión de cambiar de centro de trabajo, y que de ello derive la rotación de personal dentro de las organizaciones. Se analiza la perspectiva de diferentes autores para dar sustento teórico a la comprensión del comportamiento dentro de las organizaciones.

A partir del piloto experimental realizado en la ciudad de Nogales, Sonora, se logró identificar dentro del sector de la industria maquiladora las diferentes perspectivas de trabajadores de tres centros de trabajo con respecto a la comunicación, el liderazgo y la cultura organizacional. Entre los principales hallazgos se encuentra que el $70 \%$ de las personas son originarias de multirregiones tanto del estado de Sonora como del resto

\footnotetext{
* Doctorante en Investigación de la Comunicación por la Universidad Anáhuac México, campus Norte. Maestra en Educación Basada en Competencias por la Universidad del Valle de México. Licenciada en Comunicación Organizacional por la Universidad de Sonora. Docente de asignatura de la Universidad de Sonora, Campus Nogales, desde el 2016 a la fecha. Trabajó en la Universidad del Valle de México como docente en el área de ciencias sociales hasta el 2019.
} 
del país, lo que refleja la falta de creación de comunidad (como la unión de liderazgo individual y ciudadanía colectiva) (Mintzberg, 20I2).

Palabras clave: liderazgo, comunicación organizacional, clima organizacional, maquiladora.

\section{SUMMARY}

There is currently a high turnover of personnel within the maquiladora zone on the border of Nogales, Sonora. The focus is on identifying the perception of the operational hand of the maquiladora zone, and how organizational communication, leadership and position within the organizational structure affect the decision to change the work center, and that derives from the rotation of personnel within the organizations. The perspective of different authors is analyzed to give theoretical support to the understanding of the behavior within organizations.

From the experimental pilot carried out in the city of Nogales, Sonora within the maquiladora industry sector, we were able to identify the different perspectives of workers in three work centers with respect to communication, leadership and organizational culture. Among the main findings is that $70 \%$ of the people are from multi-regions both in the state of Sonora and the rest of the country, which reflects the lack of community building (such as the union of individual leadership and collective citizenship) (Mintzberg, 20I2).

Keywords: leadership, organizational communication, organizational climate, industry.

\section{INTRODUCCIÓN}

La comunicación es una actividad innata del ser humano, es el proceso mediante el cual se pasa información y comprensión de una persona a otra (Chiavenato, 2006). Por lo tanto, toda comunicación influye por lo menos a dos personas: el que envía el mensaje y el que lo recibe.

Dentro de un contexto actual en el cual existen ro4 fábricas maquiladoras de exportación que cuentan con una rotación promedio de 5.94\% mensual según el Consejo Nacional de la Industria Maquiladora y Manufacturera de la Exportación (INDEX), la investigación exploratoria cobra mayor relevancia porque la industria maquiladora en esta zona cuenta con un total de 45 mil I2I empleados.

Lo anterior nos lleva a analizar la comunicación interna, ya que es uno de los desafíos más importantes dentro de las organizaciones y, por tanto, como mencionaba Maslow (I99I) el rendimiento de la organización se relaciona con el interés de la gerencia sobre las 
necesidades e ideas de los trabajadores. Se revisaron las aportaciones de varios autores en el campo de la comunicación organizacional y, en particular, sobre las variables de clima y comunicación organizacional, liderazgo y condiciones laborales, motivación y compromiso laboral, pertenencia y confianza institucional.

A partir del piloto experimental realizado en la ciudad de Nogales, Sonora dentro del sector de la industria maquiladora, se logró identificar las diferentes perspectivas de trabajadores de tres centros de trabajo con respecto a la comunicación, el liderazgo y la cultura organizacional. Entre los principales hallazgos se encuentra que el $70 \%$ de las personas son originarias de multirregiones tanto del estado de Sonora como del resto del país, lo que refleja la falta de creación de comunidad (como la unión de liderazgo individual y ciudadanía colectiva) (Mintzberg, 20I2). Se comprueba que las organizaciones tienden a estructurarse buscando la armonía interna y en relación con su entorno, imitando algunas de las configuraciones identificadas por Mintzberg, sin limitarse a seguir únicamente una de ellas. Esta complejidad organizacional no permite que se genere una comunicación productiva (Nosnik, 20I3) donde se vea más allá de la retroalimentación, de modo que no hay condiciones para los cambios que se convierten en innovaciones y que mejoran el proceso de comunicación gracias a la información y al diálogo. Finalmente, se observó que los valores culturales afectan el comportamiento dependiendo del tipo de líder que los dirija (Hofstede et al., I978), y a la cultura organizacional como la programación mental colectiva que distingue a los miembros de una organización de los de otra.

Según Muriel y Rota (2005), la comunicación institucional es un sistema coordinador entre la institución y sus públicos que actúa para facilitar la consecución de los objetivos específicos de ambos y, a través de ello, contribuir al desarrollo.

Por su parte, Pérez (20I2) afirma que en la actualidad existen rasgos que evidencian los fuertes desplazamientos de la comunicación hacia los nuevos paradigmas científicos y la tendencia a la orientación de la comunicación y la estrategia hacia los seres humanos (relacionales) y los sujetos colectivos (organizacionales); sugiere además estudiar la comunicación como un fenómeno biológico y no solo social, político o económico. Si analizamos la situación actual dentro de las organizaciones, la preocupación no está concentrada en los rasgos del líder, sino en lo que hace y cómo lo hace (Palomino, 2009).

Existe amplio consenso respecto al efecto del liderazgo en el bienestar psicológico, los estados emocionales, las creencias, las conductas y el compromiso de los trabajadores, así como en el desempeño laboral y el clima organizacional (Carr et al., 2003). 
ESTADO DEL ARTE

Dentro del marco de investigación que existe sobre las variables antes mencionadas, Noboa, et al. (2019) han verificado que hay una relación positiva entre los constructos de clima organizacional y satisfacción laboral, y dentro de estas las dimensiones que tienen mayor incidencia sobre la satisfacción laboral son recompensa y liderazgo.

Díaz-Fúnez et al. (2016) establecieron que la influencia de la comunicación de funciones directivas hace disminuir el conflicto de rol y este efecto se incrementa cuando aumenta la percepción de clima de apoyo, clima de innovación y clima de orientación a reglas.

Un líder responsable es visionario y proactivo en la toma de sus decisiones, y está orientado al trabajo y las personas en su actuación. Dicho esto, la sociedad necesita de líderes responsables y esto no es una tarea fácil, debido a que se requiere transitar hacia una cultura de credibilidad y confianza en los grupos de interés (Montañez, 2015).

En otro sentido, Cavalluci (20I8) argumenta que la persona que aprende a comunicar estructurando las herramientas sabrá emprender procesos de interacción acertados en su entorno y quienes adquieren conciencia de saber gestionar estas habilidades acaban siendo líderes, heredando al mismo tiempo la responsabilidad de guiar a otros en este proceso de aprendizaje sutil y profundo. Para este autor, el líder feliz es una persona que sabe guiar al grupo, consciente de su rol favorecido en cuanto a saber manejar y gestionar los conflictos existentes, evitando que estos escalen y anticipando aquellos latentes.

Por otro lado, Viloria et al. (2016), evidencian que el liderazgo informal, como una dinámica propia de los grupos informales, puede influir de manera positiva o negativa en la productividad y competitividad al vincularla al factor humano de la organización. Concluyen que la gestión administrativa de este fenómeno organizacional se convierte en una herramienta para lograr ventajas competitivas en las organizaciones a través del mejoramiento de las variables funcionales de la empresa y el establecimiento de relaciones sociales más eficientes.

\section{MARCO TEÓRICO}

Si analizamos lo anterior, Chiavenato (2006) refiere que el liderazgo es la influencia interpersonal ejercida en una situación, que va dirigida a través del proceso de comunicación para la obtención de objetivos. Vivir en una organización, trabajar en ella, tomar parte en sus actividades y hacer carrera es participar íntimamente de su cultura.

Concretamente, se requiere reconocer cómo se relaciona el liderazgo con la rotación de personal en la zona industrial fronteriza de Nogales, Sonora, en una población de tra- 
bajadores originarios de multirregiones, así como identificar los patrones de liderazgo y comunicación que se dan dentro de las diferentes áreas. Geert Hofstede (1978), con base en estudios que realizó en la década de los 8 o involucrando a más de 50 culturas nacionales, desarrolló una teoría que explica este tipo de fenómenos. Su teoría, conocida como teoría de las dimensiones culturales, ofrece un marco para examinar cómo afectan los valores culturales al comportamiento y da pistas del porqué las personas de una cultura pueden actuar de cierta forma dependiendo del tipo de líder que los dirija. Daniel Goleman (I996), dentro de su concepto de inteligencia emocional, menciona que la aportación más importante de un líder es la creación de emociones positivas en otras personas, como pueden ser motivación, empatía, habilidades sociales, por mencionar las más evidentes.

En 1978 , Burns refería que el estudio del liderazgo es uno de los temas que más ha intrigado a los investigadores de las organizaciones durante siglos. Por ello, se requiere la actualización de las diferentes teorías que fundamenten las acciones actuales dentro de las organizaciones. Por otro lado, en su libro sobre la estructuración de las organizaciones Mintzberg (2012) menciona un concepto que sintetiza comunidad, liderazgo y ciudadanía a través de la palabra comunidad: se encuentra entre el liderazgo individual, por un lado, y la ciudadanía colectiva, por el otro. Así, cada organización puede estructurarse siguiendo distintas configuraciones. Según el mismo autor, no existen organizaciones cuya estructura corresponda completamente con una configuración. Por el contrario, las organizaciones tienden a estructurarse buscando la armonía interna y en relación con su entorno, imitando algunas de las configuraciones sin limitarse a seguir únicamente una de ellas.

Analizando el concepto de clima organizacional, Litwin y Stringer (1968) lo definen como un conjunto de propiedades medibles dentro del medio ambiente de trabajo, las cuales son percibidas directa o indirectamente por las personas que trabajan dentro de la organización, y que a su vez influyen en su motivación y comportamiento.

\section{OBJETIVOS}

Identificar las herramientas de comunicación utilizadas por los líderes organizacionales dentro del sector industrial fronterizo, y su influencia en clima laboral. Mostrar cómo inciden los aspectos psicosociales, como el sentido de pertenencia y la confianza institucional, en el compromiso laboral, en el contexto de organizaciones caracterizadas por su multiculturalidad y una determinada estructura organizacional. 


\section{METODOLOGÍA}

La investigación tiene un enfoque hermenéutico reflexivo de la investigación biográfica en donde se sustentan las aportaciones teóricas sobre la comunicación productiva de Abraham Nosnik (2013), la configuración organizacional de Henry Mintzberg (1979), la comunicación intercultural y las cinco dimensiones culturales de Geer Hofstede (I978), el bienestar integral de Daniel Kahneman y Krueger (2006), el individualismo y la colectividad de Harry Triandis (200I) y la teoría integral de Ken Wilber (1970).

A partir de diversos aportes teóricos relativos a la influencia del liderazgo en la productividad, se interpretó la influencia, el diálogo y el impacto interno de las organizaciones por medio de entrevistas a veinte operadores de producción de diferentes puntos de transportes colectivos urbanos, que se encuentran ubicados en el exterior de las instalaciones de seis fábricas de la zona industrial maquiladora de Nogales, Sonora. Además, se realizaron tres entrevistas a ingenieros de diversas áreas con diferente antigüedad en las mismas.

Se cuestionaron diferentes variables, como son: condiciones laborales, comunicación organizacional, liderazgo. Los indicadores que se trató de resaltar fueron los siguientes: prestaciones competitivas, horarios, oportunidad de crecimiento, clima organizacional, pertenencia, compromiso, aspectos psicosociales, bienestar integral. Asimismo, una dimensión abordada fue la identificación del lugar de origen del total de la muestra, lo cual nos arrojó que solo cuatro personas eran residentes de la ciudad de Nogales, Sonora.

\section{RESULTADOS}

Los principales resultados evidencian que arriba del $70 \%$ de los trabajadores provienen de multirregiones tanto del estado de Sonora como del resto del país (Gráfica I).

Observando el tiempo que tienen radicando en la ciudad fronteriza, los resultados mostraron que las personas que son foráneas actualmente tienen una estabilidad en la ciudad de Nogales de 15 años a 30 años en promedio (Gráfica 2). El tiempo que tienen residiendo en Nogales es el mismo tiempo que llevan laborado en el sector maquiladora. 
PRINCIPALES HALLAZGOS

- Falta comunicación con líderes. Se manifestó en repetidas ocasiones que los líderes son los que en algún momento fueron operadores y actualmente no tienen manejo de liderazgo (ordenan).

- Se tiene una relación cordial entre todos los compañeros, pero hay mucho choque de personalidad (Gráfica 3).

- Las personas que trabajan en las áreas operativas cuentan con más de tres años de trabajo; sí consideran cambiar de trabajo, siempre y cuando sea por mejor salario.

- Más del 80\% manifiesta haber tenido algún problema de comunicación con su jefe directo (Gráfica 4).

- La mayoría de las personas que son foráneas se encuentran en la frontera norte porque aquí les ofrecen mejores salarios (Gráfica 5).

- Más de tres operadores manifestaron deseos de superación personal (académica y laboral).

- El medio que más utilizan los superiores para dirigirse a los operadores es la comunicación verbal (Gráfica 6).

- La mayoría manifestó que hay un buen ambiente de trabajo, sin importar problemas de comunicación que ocasionalmente surgen.

- Los ingenieros no piensan en cambiar de trabajo en el corto plazo, pero sí en un futuro.

- A las personas de otras ciudades o estados se les trata diferente, con un trato que incluye carrilla, y cuando hay visitas foráneas o extranjeras siempre dicen que son gringos.

- Los ingenieros se manifiestan satisfechos.

- Los operadores se manifiestan conformes. 


\section{GRÁFICA 1. LUGAR DE ORIGEN}

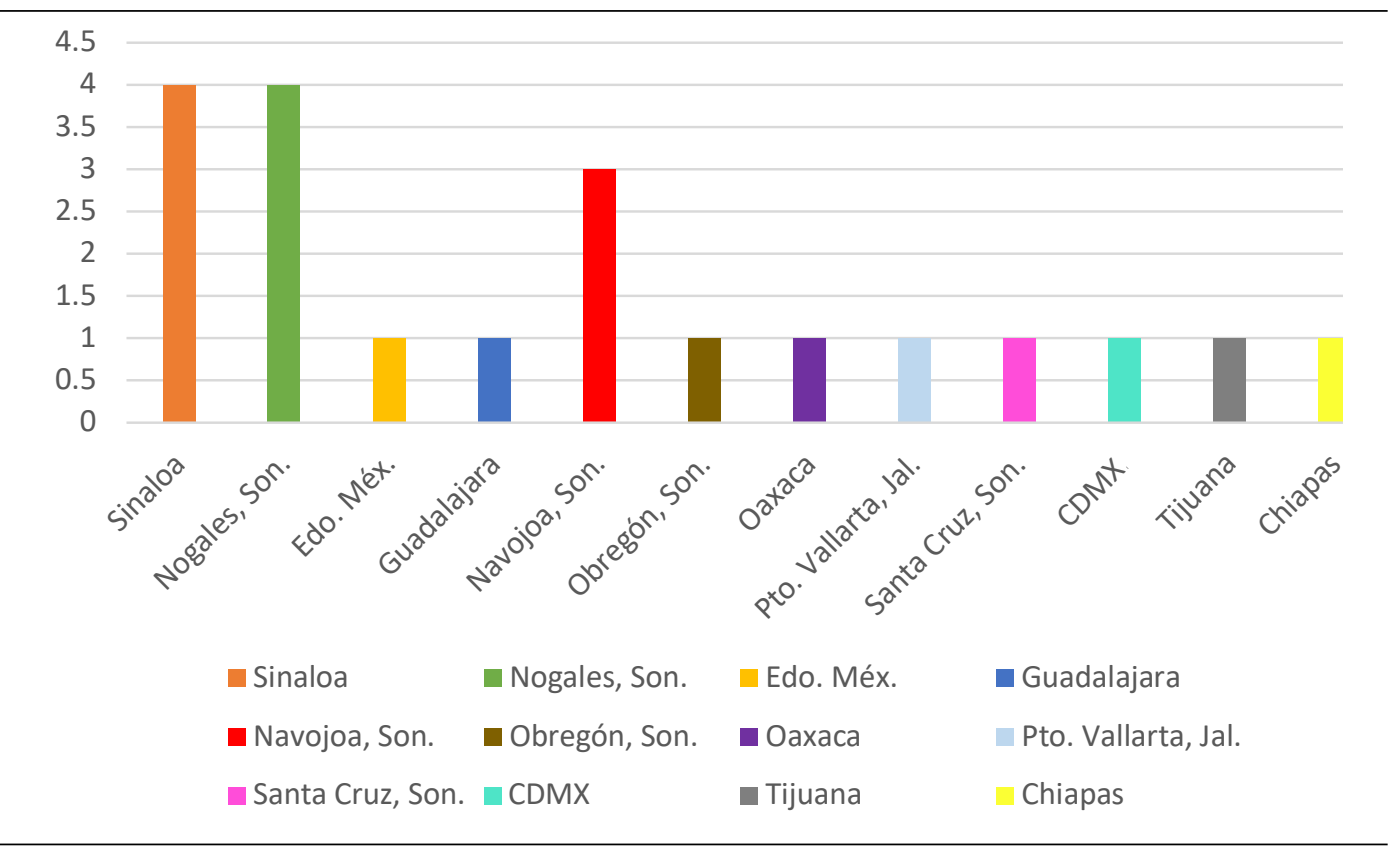

FUENTE: elaboración propia.

\section{GRÁFICA 2. AÑOS DE RESIDENCIA EN LA CIUDAD DE NOGALES}

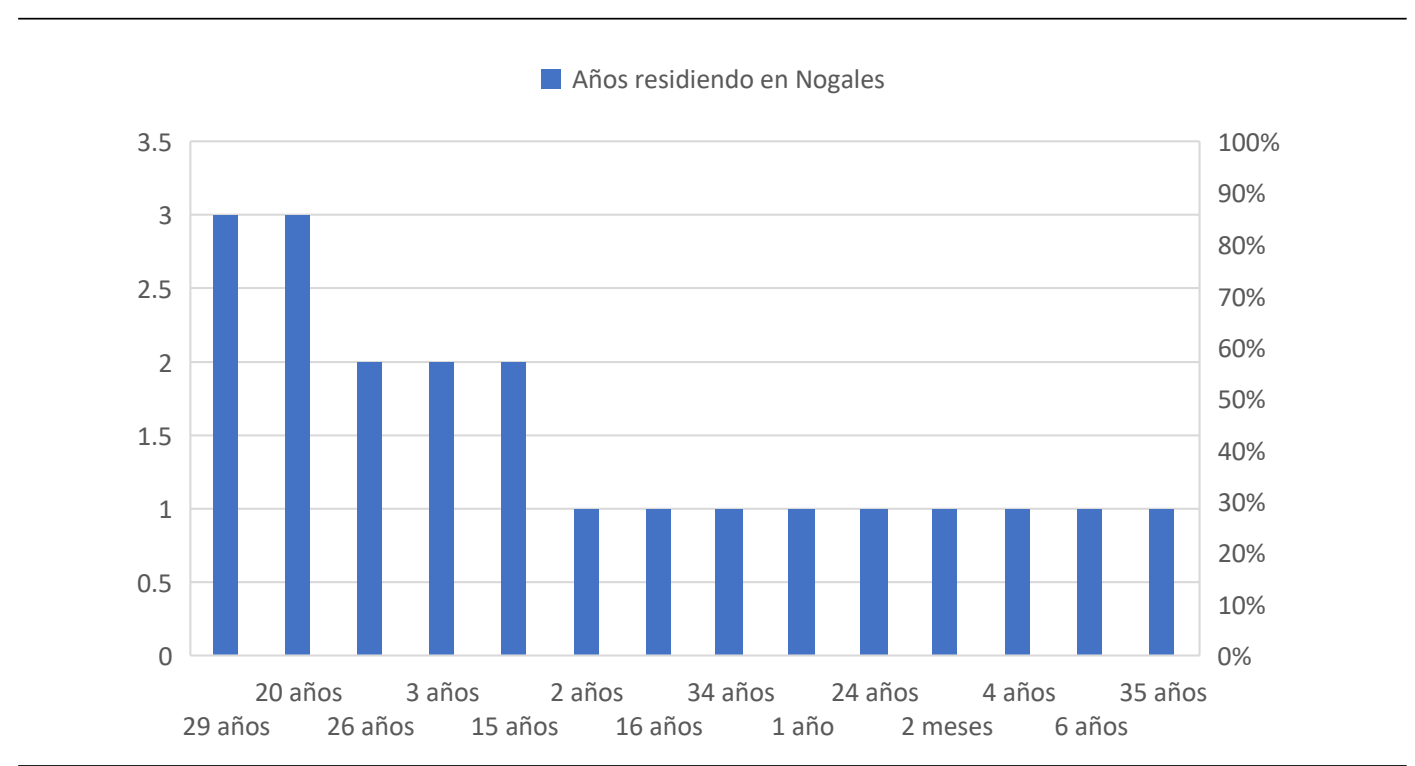

FUENTE: elaboración propia. 


\section{GRÁFICA 3. PERCEPCIÓN SOBRE LAS RELACIONES LABORALES}

¿Son positivas las relaciones entre todos los trabajadores?

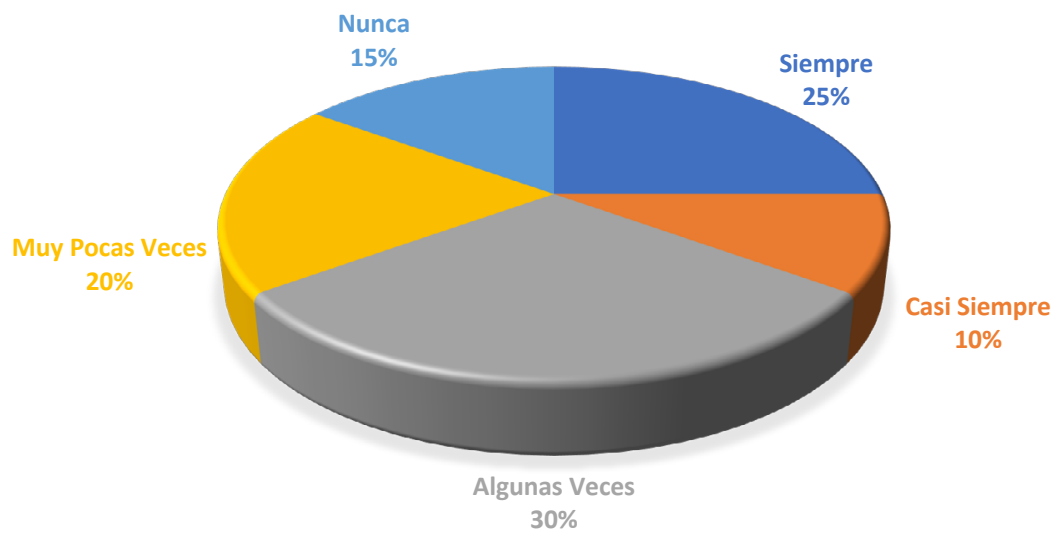

FUENTE: elaboración propia.

\section{GRÁFICA 4. COMUNICACIÓN Y LIDERAZGO}

¿Ha tenido algún problema de comunicación con su líder directc

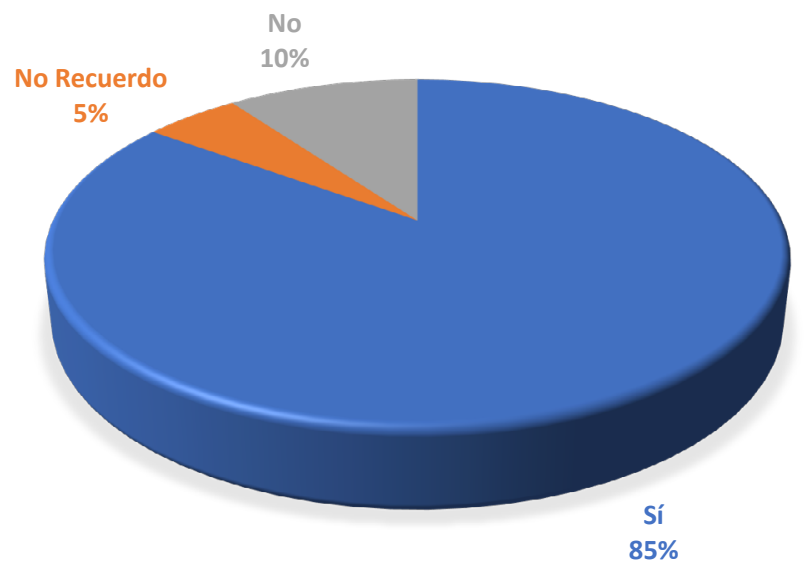

FUENTE: elaboración propia. 


\section{GRÁFICA 5. IDENTIFICACIÓN DE CAUSAS DE ROTACIÓN LABORAL}

¿Cuál es el principal factor que lo ha orillado a cambiar de centro de trabajo?

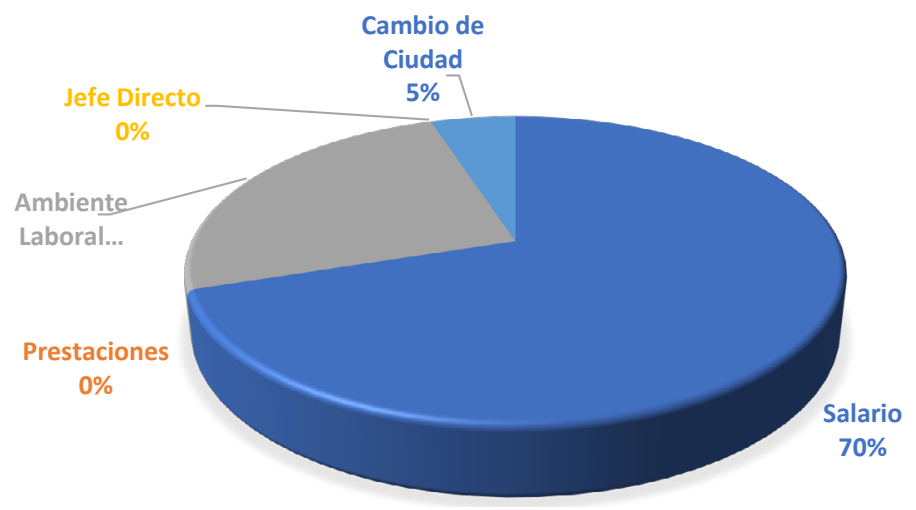

FUENTE: elaboración propia.

\section{GRÁFICA 6. MEDIOS DE COMUNICACIÓN INTERNA}

¿Cuál es el medio de comunicación que más utiliza su superior para dirigirse a usted?

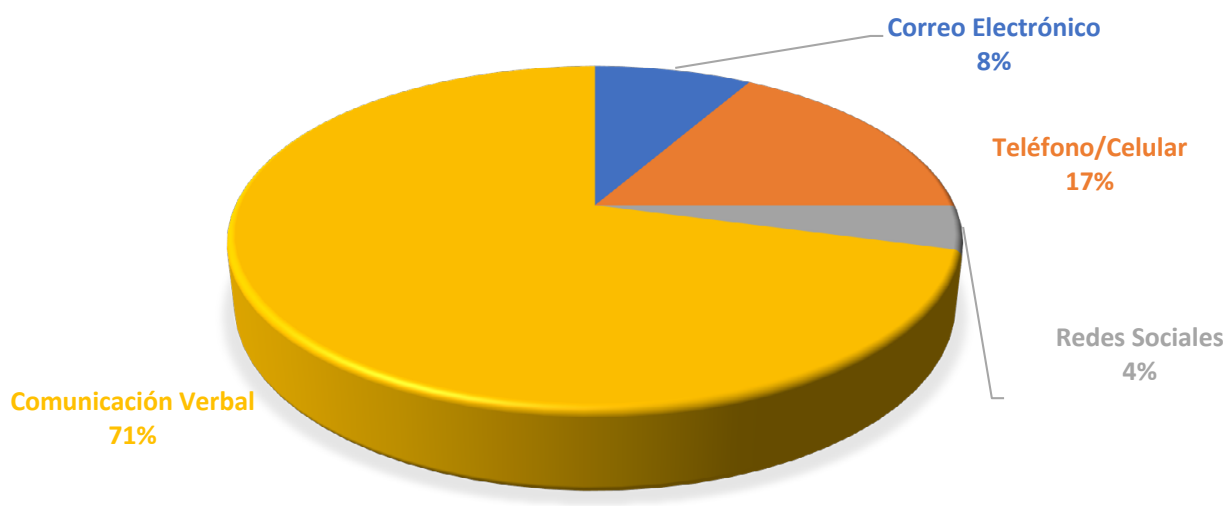

FUENTE: elaboración propia. 


\section{DISCUSIÓN}

La perspectiva que tiene cada uno de los trabajadores del sector maquiladora respecto a la experiencia dentro de su área de trabajo es diversa, ya que todos han manifestado diferentes roles: desde ser personas a las cuales se les ha tratado como foráneos o con carrilla, hasta personas que manifiestan no haber tenido ningún inconveniente. Sin embargo, la mayoría expone que los problemas de comunicación siempre han existido, ya que en ocasiones la manera de solicitar las cosas o los métodos que utilizan no son los más eficaces.

Los líderes que actualmente se encuentran al mando son personas que ascendieron de puestos operativos y hoy ocupan posiciones como supervisores y/o líderes de línea. En la encuesta eso se muestra como un factor de falta de formación, ya que se les calificó de líderes prepotentes o soberbios al momento de dar una indicación.

Es interesante hacer la comparación con las entrevistas realizadas a personas de puestos de mando alto, que son los ingenieros de área. Estos indican no tener ningún problema de comunicación; sin embargo, refieren no tener mayores oportunidades de crecimiento dentro de su centro de trabajo y esta sería la causa principal de un posible cambio trabajo.

Se concluye que la empatía, el tacto y la sensibilización de los líderes en diversas áreas se muestran como factores primordiales para un desarrollo eficaz y, a su vez, para generar un ambiente de trabajo grato. Esto permitiría crear un compromiso organizacional en que los trabajadores se sientan respaldados, se aliente la satisfacción en el trabajo y la responsabilidad laboral, disminuyendo con ello la rotación de puestos.

\section{REFERENCIAS}

Aguilera, J. (2007). Comunicación organizacional contemporánea. El aquí y el abora de la comunicación en la empresa. https://www.gestiopolis.com/evolucion-comunicacion-organizacional/

Barahona, H., Cabrera, D. R., y Torres, U. E. (20II). Los líderes en el siglo XXI. Entramado, 7(2), 86-97. http://dialnet.unirioja.es/servlet/articulo?codigo $=3942518$

Blake, R., y Mouton, J. (1985) The managerial grid: the key to leadership excellence. Houston, TX: Gulf Publishing Company.

Burns, J. M. (1978). Leadership. Harper y Row, New York.

Calderón, C., Mendoza, J. C. (2000). Demanda regional de trabajo de la industria maquiladora de exportación en los estados de la frontera norte. Frontera Norte, I3(24). https://www.redalyc.org/ articulo.oa?id $=13602403$

Carr,J, Schmidt, A, Ford, J.,y De Shon, R. (2003). Climate perceptions matter: a meta-analytic path analysis relating molar climate, cognitive and affective states, and individual level work outcomes. Fournal of Applied Psychology, 88(4), 605-619. https://doi.org/10.1037/002I-9010.88.4.605 
Carro-Suárez, J., Sarmiento-Paredes, S., y Rosano-Ortega, G. (2017). La cultura organizacional y su influencia en la sustentabilidad empresarial. la importancia de la cultura en la sustentabilidad empresarial. Estudios Gerenciales, 33(I45), 352-365.http://dx.doi.org/10.IoI6/j.estger.2017.II.006

Cavallucci, O. (2018). El reto de la comunicación: liderazgo comunicacional y herramientas negociadoras. Revista Estudios de la Gestión: Revista Internacional de Administración, (I), II9-I35. http://167.I72.193.213/index.php/eg/article/view/573

Consejo Empresarial Nogales, A. C. http://consejoempresarial.org/

Cornejo-Aparicio, V., y Flores-Silva, S. (2020). Modelo para el diseño organizacional basado en cualidades. Revista Ibérica de Sistemas e Tecnologias de Informação, 733-750. https://search.proquest.com/openview/8a2868ccf43245beo094007eor 479 d5a/I.pdf?pq-origsite=gscholar\&$\mathrm{cbl}=1006393$

Coronado, E. (2020, Io de abril). ARINAC (Asociación de Relaciones Industriales de Nogales, A. C.): Una historia oral. Entrevista al presidente de AR INAC por Mirla Yessenia Castro Rodríguez.

Chiavenato, I. (2006). Introducción a la teoría general de la administración. McGraw-Hill Interamericana.

Díaz-Fúnez, P. A., Mañas-Rodríguez, M. Á., Pecino-Medina, V., Salvador- Ferrer, C. M., \& Llopis-Marín, J. M. (20I6). El efecto modulador del clima organizacional en la influencia de la comunicación de funciones directivas sobre el conflicto de rol. Universitas Psychologica, I5(I), 15-25. https://doi.org/I0.III44/Javeriana.upsyi5-I.emco

Galhardi, R. (1998). Maquiladoras prospects of regional integration and globalization. Employment and Training Papers, I2, Organización Internacional del Trabajo. www.ilo.org/public/english/ employment/strat/publ/etpi2.htm

García-Solarte, M. (2015). Formulación de un modelo de liderazgo desde las teorías organizacionales. Entramado, II(I), 60-79. http://dx.doi.org/I0.1804I/entramado.20I5viInI.2IIII

Goleman, D. (1996). La inteligencia emocional. Madrid: Kairós.

Hofstede, G., Hofstede, G. J., y Minkov, M. (1978). Cultures and organizations: software of the mind. McGraw-Hill.

INDEX (Consejo Nacional de la Industria Maquiladora y Manufacturera de la Exportación). https://www.index.org.mx/index.html

Kahneman, D., Krueger, A.B. (2006). “Avances en la Medición del Bienestar Subjetivo”. Revista de perspectivas económicas, 20 (I), 3-24

Litwin G. H., y Stringer, R. A. (1968). Motivation and organizational climate. Harvard University Press.

Maslow, A. (199I). Motivación y personalidad. Ediciones Díaz de Santos.

Mindtools (s.f.). The Blake Mouton managerial grid: leading people and producing results. https://www. mindtools.com/pages/article/newLDR_73.htm

Mintzberg, H. (2012). La estructuración de las organizaciones. Ariel.

Montañez, G. (2015). Hacia el perfil del liderazgo responsable empresarial. Revista Iberoamericana de Contaduría, Economía y Administración, 4(7), I36-I48.

Muriel, M., y Rota, G. (2005). Comunicación institucional: enfoque social de relaciones públicas. Ediciones Ciespal. 
Murillo, C. (20I4). Regionalismo e integración regional: una aproximación teórica. Revista Centroamericana de Administración Pública, (66/67), I83-2II. https://doi.org/10.35485/rcap66/67_6

Noboa, J. G., Barrera, G. J., y Rojas, D. (2019). Relación del clima organizacional con la satisfacción laboral en una empresa del sector de la construcción. Revista Científica Ecociencia, 6(I), I-24. https://doi.org/I0.2I855/ecociencia.61.184

Nosnik, A. (2012). Teoría de la comunicación productiva: exploraciones más allá de la retroalimentación. Homo Sapiens.

Nosnik, A., y Martínez, A. (1988). Comunicación organizacional práctica: manual gerencial. Trillas.

Palomino, P. R. (2009). Últimas tendencias en el estudio sobre liderazgo: revisión de la literatura. Documentos de Trabajo. Seminario Permanente de Ciencias Sociales, (4), I-I6.

Pérez, R. A. (2012). El estado del arte en la Comunicación Estratégica. Mediaciones Sociales, (Io), I2I-I96. https://doi.org/I0.5209/rev_MESO.20I2.nIo.39684

Pupo, B. (20I8). Contribución de la gestión del clima organizacional al fomento de valores organizacionales y al logro del desempeño individual. Examen de un caso de estudio.

Revista Dilemas Contemporáneos: Educación, Política y Valores, 6(I). https://www.dilemascontemporaneoseducacionpoliticayvalores.com/index.php/dilemas/article/view/8I5

Rebeil, M.A.(2014). Para una comprensión de la teoría práctica de la comunicación. Razón y palabra. Investigación en Comunicación Aplicada, (84).

Rey, J., Caro, F. J., y Balhadj, S. (20I7). Los retos de la comunicación intercultural en la era de la globalización: el caso de las empresas españolas ubicadas en Marruecos. Prisma Social, (I7), 415-437. https://revistaprismasocial.es/article/view/I29I

Rodríguez-Fernández, L., y Vázquez-Sande, P. (2019). Retos y perspectivas en la comunicación organizacional. El Profesional de la Información, 28(5). http://dx.doi.org/I0.3145/epi.2019.sep.oI

Taylor, D. L. (2003). Los orígenes de la industria maquiladora en México. Comercio Exterior, 53(II).

Tratado de Libre Comercio de América del Norte. http://idatd.cepal.org/Normativas/TLCAN/ Espanol/Tratado_de_Libre_Comercio_de_America_del_Norte-TLCAN.pdf

Triandis, H. C. (I990). Aproximaciones teóricas y metodológicas al estudio del individualismo y colectivismo. Revista de Psicología Social y Personalidad, 6(I-2), 29-38.

Unesco (2010). Invertir en la diversidad cultural y el diálogo intercultural: informe mundial de la Unesco. Unesco. https://unesdoc.unesco.org/ark:/48223/pfoooor 87828

Vargas, M. R. (2003). Industria maquiladora de exportación: ¿Hacia dónde va el empleo? Papeles de población, 9(37), 243-259. http://www.scielo.org.mx/scielo.php?script=sci_arttext\&pi-

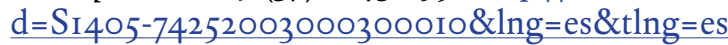

Vargas, L. (2000). Maquilas 2000: Still Growing. Business Frontier, (3). https://www.dallasfed. org/ /media/documents/research/busfront/busooo3.pdf

Viloria-Escobar,J. de J., Pedraza-Álvarez,L.P., Cuesta-Tamayo, K., y Pérez-Correa, K. (20I6).Liderazgo informal en las organizaciones: Análisis sobre su impacto e influencia en la productividad y competitividad. Clío América, Io(I9), 31-42. https://doi.org/I0.21676/23897848.1658

Wilber, K. (1977). El ojo del espíritu. Una visión integral para un mundo que está enloqueciendo poco a poco. Barcelona: Kairós.

Esta obra está bajo Licencia Creative Commons Atribución-NoComercial-SinDerivadas 4.o Internacional. 